\title{
Trauma Surgery $\&$ Acute Care Open \\ Current outcomes of blunt open pelvic fractures: how modern advances in trauma care may decrease mortality
}

\author{
Sammy S Siada, James W Davis, Krista L Kaups, Rachel C Dirks, Kimberly A Grannis
}

Department of Surgery, Community Regional Medical Center, University of California, San Francisco-Fresno, Fresno, California, USA

Correspondence to Dr Sammy S Siada, Department of Surgery, Community Regional Medical Center, Fresno, CA 93721, USA; ssiada@fresno. ucsf.edu

This paper was presented as a poster at the 75th annual meeting of the American Association for the Surgery of Trauma, Sep 2016.

Received 23 October 2017 Revised 22 November 2017 Accepted 27 November 2017

To cite: Siada SS, Davis JW, Kaups KL, et al. Trauma Surg Acute Care Open 2017:2:1-4.

\begin{abstract}
Background Open pelvic fracture, caused by a blunt mechanism, is an uncommon injury with a high mortality rate. In 2008, evidence-based algorithm for managing pelvic fractures in unstable patients was published by the Western Trauma Association (WTA). The use of massive transfusion protocols has become widespread as has the availability and use of pelvic angiography. The purpose of this study was to evaluate the outcome of open pelvic fractures in association with related advances in trauma care.

Methods A retrospective review was performed, at an American College of Surgeon verified level I trauma center, of patients with blunt open pelvic fractures from January 2010 to April 2016. The WTA algorithm, including massive transfusion protocol, and pelvic angiography were uniformly used. Data collected included injury severity score, demographic data, transfusion requirements, use of pelvic angiography, length of stay, and disposition. Data were compared with a similar study from 2005.

Results During the study period, 1505 patients with pelvic fractures were analyzed; 87 (6\%) patients had open pelvic fractures. Of these, 25 were from blunt mechanisms and made up the study population. Patients in both studies had similar injury severity scores, ages, Glasgow Coma Scale, and gender distributions. Use of angiography was higher (44\% vs. $16 \% ; P=0.011)$ and mortality was lower ( $16 \%$ vs. $45 \% ; P=0.014$ ) than in the 2005 study.

Conclusions Changes in trauma care for patients with open blunt pelvic fracture include the use of an evidence-based algorithm, massive transfusion protocols and increased use of angioembolization. Mortality for open pelvic fractures has decreased with these advances.

Level of evidence Level IV.
\end{abstract}

\section{BACKGROUND}

Open pelvic fracture is a morbid injury and very often is a lethal one. Historically, mortality rates have been reported to range from 5\% to as high as $50 \% \cdot{ }^{1-4}$ Early mortality is related to exsanguinating hemorrhage and late mortality is generally due to pelvic sepsis.

In 2005, Dente and colleagues retrospectively analyzed patients who sustained open pelvic fractures as a result of blunt mechanism between 1995 and 2004. ${ }^{5}$ The overall mortality in that series was $45 \%$. Since that study was published, there has been a paucity of published data on the outcomes of open pelvic fractures despite significant advancements in the care of these injuries.

The Western Trauma Association (WTA) published an algorithm for Management of Pelvic Fractures with Hemodynamic Instability in 2008, which provided an evidence-based schema for this injury. ${ }^{6}$ The algorithm emphasizes the importance of a multidisciplinary approach with trauma surgery, orthopedics, and interventional radiology. It also addresses diagnostic evaluation, exclusion of intra-abdominal injury, pelvic stabilization, and decisions concerning surgical options and angiography.

The management of coagulopathy has also evolved since the study by Dente et al was published. Implementation of a massive transfusion protocol (MTP) with 1:1:1 ratio of packed red blood cells (PRBC) to fresh frozen plasma (FFP) to platelets has been shown to reduce mortality in patients with traumatic hemorrhage. ${ }^{7-9}$ This is due to expeditious product availability and aggressive transfusion of blood products that enables quick restoration of intravascular volume and treatment of coagulopathy.

Additionally, the use of pelvic angiography and embolization has increased in the last decade and has been found to be an effective adjunct for control of pelvic fracture hemorrhage. ${ }^{10}$ Another advancement has been the advent of hybrid operating rooms (ORs), which are increasingly being used in trauma. ${ }^{11}$

The purpose of this study was to examine outcomes for blunt open pelvic fractures in the current era and compare them with previously reported data. We hypothesize that the changes in care of patients with open pelvic fractures have led to a decreased overall mortality.

\section{METHODS}

A retrospective review was performed on patients admitted to Community Regional Medical Center (CRMC), an ACS-verified level 1 trauma center, from January 1, 2010 through April 30, 2016.

Patients with open pelvic fractures from blunt mechanism were identified from the trauma registry and were included in this study. Data collected included the following: age, sex, injury severity score (ISS), ${ }^{12}$ Glasgow Coma Scale (GCS), Gustilo grade of soft tissue injury, orthopedic management, use of pelvic angiography, operative management, hospital length of stay (LOS), transfusion requirements in the first 24 hours, and final disposition. 


\begin{tabular}{ll} 
Table 1 & Gustilo-Anderson's classfication \\
\hline Type & Definition \\
\hline I & Clean wound, $<1 \mathrm{~cm}$ in length \\
& $>1 \mathrm{~cm}$ but $<10 \mathrm{~cm}$ in length without extensive soft tissue \\
II & damage, flaps, or avulsions \\
III & $>10 \mathrm{~cm}$ in length, fractures open for $>8$ hours \\
\hline
\end{tabular}

Patients with penetrating injuries and closed fractures were excluded.

Patients were initially evaluated in the emergency department (ED) with a collaborative team of in-house residents, fellows, and attending physicians from the departments of trauma surgery and emergency medicine. The WTA algorithm is the guideline by which patients with pelvic fractures are treated at CRMC. The initial diagnosis of pelvic fracture was made by a pelvic X-ray obtained during the primary survey. Hemodynamically stable patients were taken to the CT scanner for an abdominopelvic scan and if contrast extravasation in the pelvis was visualized, the patient was sent for pelvic angiographic and possible embolization. The MTP was initiated in patients who were hemodynamically unstable (any systolic blood pressure less than 90). A focused assessment with sonography for trauma (FAST) or diagnostic peritoneal lavage was performed to exclude intra-abdominal injury. Patients were taken to the OR for laparotomy if the FAST was positive and were sent for angiography if the FAST was negative. Pelvic binders were placed when patients have open book fractures and were hypotensive.

FAST was readily available and used when indicated. Pelvic stabilization in the ED, when performed, involved the use of a commercially available external pelvic stabilizer. A MTP designed to optimize the goal of a $1: 1: 1$ PRBC to FFP to platelet ratio was uniformly used. There was 24 hours availability of pelvic angiography, which was performed in the angiography suite or hybrid OR by an interventional radiologist.

The degree of soft tissue injury was defined using the Gustilo-Anderson and Faringer classifications (tables 1 and 2). ${ }^{13} 14$ The data obtained in this study were compared with the 2005 study by Dente and colleagues. Using the 2005 study as a historical control group, comparisons were made between the patient demographics, ISS, LOS, injury severity and classification, use of fecal diversion, and overall mortality rate. Dichotomous variables were compared using $\chi^{2}$ analysis with a significance attributed to a $P$ value of $<0.05$. Data were analyzed using SPSS V.23.0.

\section{RESULTS}

During the study period, 1505 patients had pelvic fractures, with $87(6 \%)$ classified as open. Of these, 25 were from blunt mechanisms of injury and made up the study population. The average age was 42 years and $68 \%$ of patients were men. The mean ISS was 29 and the mean hospital LOS in survivors was 21 days. Age, ISS, GCS, and length of stay were similar between this study and the 2005 study (table 3). Fifteen patients in this series were

\begin{tabular}{ll}
\hline Table 2 & Faringer soft tissue injury classification \\
\hline Grade & Wound \\
\hline I & Perineal, perirectal, vaginal, posterior sacrum \\
II & Medial thigh, groin crease \\
III & Iliac crest, buttock \\
\hline
\end{tabular}

\begin{tabular}{lllll} 
Table 3 & \multicolumn{1}{l}{ Baseline demographic data } & & \\
\hline & ISS & GCS & Age (years) & Males \\
\hline 2005 & 30 & 12 & 39 & $68 \%$ \\
2016 & 29 & 11 & 42 & $68 \%$ \\
P value & 0.87 & 0.67 & - & 0.98
\end{tabular}

GCS, Glasgow coma scale; ISS, injury severity score.

admitted to the intensive care unit (ICU) and had an average ICU length of stay of 13 days.

The most common Gustilo grade of soft tissue injury was grade III which occurred in 10 patients (40\%) followed by grade II in eight patients (32\%) and grade I in seven patients (28\%). The most common Faringer grade of injury was grade III, occurring in 16 patients (64\%), followed by grade I in six patients $(24 \%)$ and grade II in five patients (20\%). There was no statistical difference in the frequency of grades I, II, and III soft tissue injuries between this series and the Dente series (table 4).

In the study population, 14 patients $(56 \%)$ were hypotensive in the first 24 hours of admission, and 11 had MTP initiated. The remaining three patients stabilized after transfusion of two units of PRBC. Additionally, $68 \%$ of patients required transfusion of PRBC with eight patients (32\%) requiring 10 or more units of PRBC. There was no difference in the rate of PRBC transfusion between this study and the 2005 study $(\mathrm{P}=0.68)$.

When MTP was initiated, patients received an average of 15 units of PRBC, nine units of FFP, and two units of apheresis platelets. Average international normalized ratio (INR) at admission was 1.4 in all patients and 1.8 in patients who had MTP. Use of FFP and platelets and INR were not reported in the 2005 study.

Eleven patients underwent angiography and 10 patients had embolization performed. Seven of the angiograms showed active extravasation of contrast and non-selective embolization was performed with Gelfoam (Pfizer Inc., New York, NY). Prophylactic non-selective embolization of the internal iliac arteries was performed in the remaining three patients. Overall, the embolization rate of $40 \%$ in this study was significantly higher than the rate of $16 \%$ reported in the 2005 study $(\mathrm{P}=0.026)$ (table 5$)$. Eight of the 10 patients who underwent angioembolization went directly from the ED to the angiography suite in an average time of 2.1 hours from arrival to the ED.

Five patients (20\%) underwent laparotomy within 24 hours. Fecal diversion was performed in four patients due to rectal injury or severe perineal laceration. There was no difference in the rate of fecal diversion between this study and the 2005 study $(\mathrm{P}=0.70)$.

Overall mortality rate in this study was $16 \%$ (4 patients), which was significantly lower than the $45 \%$ reported in the 2005 study $(\mathrm{P}=0.014)$ (table 5). The average ISS of the patients who died was 37 . Three of the deaths occurred in the first 24 hours and were due to exsanguinating hemorrhage, one death occurred on hospital day 90 due to multiple strokes and multisystem organ dysfunction. This patient remained in the hospital for serial

\begin{tabular}{lccl}
\hline Table 4 & Distribution of Gustilo-Anderson's grade of injury & \\
\hline Grade & $\begin{array}{l}\text { No. of patients from } \\
\text { 2005 study (\%) }\end{array}$ & $\begin{array}{l}\text { No. of patients from } \\
\text { 2016 study (\%) }\end{array}$ & P value \\
\hline I & $9(20)$ & $7(28)$ & 0.48 \\
II & $16(36)$ & $8(32)$ & 0.72 \\
II & $19(43)$ & $10(40)$ & 0.80 \\
\hline
\end{tabular}


Table 5 Comparison of treatments and mortality between 2005 and 2016

\begin{tabular}{llllll}
\hline Study $(\mathbf{n})$ & Patients transfused & Pelvic embolization & Fecal diversion & Length of stay & Mortality \\
\hline $2005(44)$ & $32(73 \%)$ & $7(16 \%)$ & $4(9 \%)$ & 22 & $20(45 \%)$ \\
$2016(25)$ & $17(68 \%)$ & $10(40 \%)$ & $3(12 \%)$ & 21 & $4(16 \%)$ \\
P value & 0.68 & 0.026 & 0.70 & - & 0.014 \\
\hline
\end{tabular}

washout and debridement of a complex pelvic wound and developed multiple strokes after one of the debridements.

One patient was transferred to another hospital after a 24-day LOS in the ICU. The remainder of survivors were eventually discharged home $(n=7)$, to a skilled nursing facility $(n=6)$, or to a rehabilitation center $(n=7)$. In the 2005 study, nearly half of the deaths occurred after 24 hours, an average of 17 days after injury and were due to pelvic sepsis, traumatic brain injury, respiratory arrest, buttock necrosis, and pulmonary embolism. None of the patients in this series died of these causes.

\section{DISCUSSION}

Open pelvic fracture by blunt mechanism is a relatively rare injury that has a high mortality rate. The purpose of this study was to investigate advances in trauma care for this injury and how mortality may have changed. When compared with a previous study of blunt open pelvic fractures, the patient demographics were similar; however, the mortality rate for open pelvic fracture decreased significantly from $45 \%$ to $16 \%$. In a single institution study that assessed the impact of damage control resuscitation (ie, MTP with 1:1:1 ratios) on pelvic ring fractures, Fitzgerald and colleagues also found an improvement in the mortality of open pelvic fractures to $16 \% .^{15}$

This decrease in mortality may be attributed to the advances in the care of patients with pelvic fractures. Implementation of a standardized algorithm has allowed for uniform and streamlined care. The development of MTP has led to more efficient and effective treatment of coagulopathy as MTPs did not exist at the time of Dente et al's study. Additionally, pelvic angiography is more liberally used today than it was in 2005. In the 2005 study, all nine patients who underwent embolization ultimately died, whereas in this study, only two patients out of the 10 died. This is likely due to the widespread use of the standardized algorithm which advocates for early angiography when appropriate in addition to better resuscitation with MTP. The average time from arrival to the ED to transfer to the angiography suite was 2.1 hours.

One weakness that was reported in the 2005 study was that there was no specific protocol to manage open pelvic fractures. Since that study was published, the WTA published an algorithm for managing pelvic fractures in 2008, simplifying the decision-making process for this severe injury. This algorithm has been used to guide management at our institution since its publication.

The assessment and treatment of coagulopathy has also evolved during the past decade. A MTP was adopted at our institution in 2008. It can be initiated by any provider involved in the care of a patient with trauma and is usually started early in the resuscitation process. PRBCs, FFP, and platelets are transfused rapidly in a 1:1:1 ratio. Coagulation studies were not reported in the 2005 study; however, 25\% of the patients died within 24 hours of arrival to the hospital due to exsanguinating hemorrhage. Only $12 \%$ of patients in this series died of exsanguination in the first 24 hours $(\mathrm{P}=0.2)$.
Although preperitoneal packing is included in the WTA algorithm, no patients in the present series underwent preperitoneal pelvic packing. Preperitoneal pelvic packing is less likely to be effective with open pelvic fractures because the tamponade of the retroperitoneum is already released to the external environment, particularly in patients with perineal wounds. ${ }^{16}$ However, early pelvic packing in combination with external fixation has been shown to decrease mortality for hemodynamically unstable patients with severe pelvic ring disruptions. ${ }^{17}$

Fecal diversion has been highlighted in the literature as a useful adjunct to prevent pelvic sepsis, especially in patients with rectal injuries. ${ }^{18-20}$ Use of fecal diversion in the present study population was not different from what has been described in previous studies. As anticipated, it was used predominately in patients with rectal injuries and those with severe perineal lacerations to avoid contamination.

This study is heir to all the limitations of retrospective reviews. Additionally, the relative scarcity of this injury limits the size of the study population. The 2005 report does not note the management of coagulopathy or the patient condition at time to angiography. These could be additional factors that influence outcomes as well. Lastly, only patients as far back as January 2010 were assessed due to lack of electronic medical records before that time.

In conclusion, the care for patients sustaining open pelvic fractures by blunt mechanism has evolved in recent years. Changes include the use of an evidence-based algorithm, better treatment of coagulopathy including MTPs, and increased use of angioembolization. The overall mortality for open pelvic fractures has decreased with these advances.

Contributors SSS and JWD contributed to study design, data collection, data analysis, data interpretation, writing, and critical revision. KLK contributed to data interpretation and critical revision. RCD contributed to data collection, data analysis, and data interpretation. KAG contributed to literature search and data collection

Competing interests None declared.

Ethics approval UCSF Fresno and Community Medical Centers IRB.

Provenance and peer review Not commissioned; externally peer reviewed.

Open Access This is an Open Access article distributed in accordance with the Creative Commons Attribution Non Commercial (CC BY-NC 4.0) license, which permits others to distribute, remix, adapt, build upon this work non-commercially, and license their derivative works on different terms, provided the original work is properly cited and the use is non-commercial. See: http://creativecommons.org/ licenses/by-nc/4.0/

(c) Article author(s) (or their employer(s) unless otherwise stated in the text of the article) 2017. All rights reserved. No commercial use is permitted unless otherwise expressly granted.

\section{REFERENCES}

1 Richardson JD, Harty J, Amin M, Flint LM. Open pelvic fractures. J Trauma 1982;22:533-8.

2 Rothenberger D, Velasco R, Strate R, Fischer RP, Perry JF. Open pelvic fracture: a lethal injury. J Trauma 1978;18:184-7.

3 Jones AL, Powell JN, Kellam JF, McCormack RG, Dust W, Wimmer P. Open pelvic fractures. A multicenter retrospective analysis. Orthop Clin North Am 1997;28:345-50. 
4 Hanson PB, Milne JC, Chapman MW. Open pelvis fractures. Review of 43 cases. $J$ Bone Joint Surg Br 1991;73:325-9.

5 Dente CJ, Feliciano DV, Rozycki GS, Wyrzykowski AD, Nicholas JM, Salomone JP, Ingram WL. The outcome of open pelvic fractures in the modern era. Am J Surg 2005;190:831-7.

6 Davis JW, Moore FA, McIntyre RC, Cocanour CS, Moore EE, West MA. Western trauma association critical decisions in trauma: management of pelvic fracture with hemodynamic instability. J Trauma 2008:65:1012-5.

7 Riskin DJ, Tsai TC, Riskin L, Hernandez-Boussard T, Purtill M, Maggio PM, Spain DA Brundage SI. Massive transfusion protocols: the role of aggressive resuscitation versus product ratio in mortality reduction. J Am Coll Surg 2009;209:198-205.

8 Malone DL, Hess JR, Fingerhut A. Massive transfusion practices around the globe and a suggestion for a common massive transfusion protocol. I Trauma 2006;60(6 Suppl):S91-6.

9 Dente CJ, Shaz BH, Nicholas JM, Harris RS, Wyrzykowski AD, Patel S, Shah A Vercruysse GA, Feliciano DV, Rozycki GS, et al. Improvements in early mortality and coagulopathy are sustained better in patients with blunt trauma after institution of a massive transfusion protocol in a civilian level I trauma center. I Trauma 2009:66:1616-24.

10 Fu CY, Wang YC, Wu SC, Chen RJ, Hsieh CH, Huang HC, Huang JC, Lu CW, Huang YC. Angioembolization provides benefits in patients with concomitant unstable pelvic fracture and unstable hemodynamics. Am J Emerg Med 2012;30:207-13.

11 Fehr A, Beveridge J, D’Amours SD, Kirkpatrick AW, Ball CG. The potential benefit of a hybrid operating environment among severely injured patients with persistent hemorrhage: how often could we get it right? J Trauma Acute Care Surg 2016:80:457-60.

12 Baker SP, O'Neill B, Haddon W, Long WB. The injury severity score: a method for describing patients with multiple injuries and evaluating emergency care. J Trauma 1974;14:187-96.

13 Gustilo RB, Anderson JT. Prevention of infection in the treatment of one thousand and twenty-five open fractures of long bones. J Bone Joint Surg Am 1976:58:453-8.

14 Faringer PD, Mullins RJ, Feliciano PD, Duwelius PJ, Trunkey DD. Selective fecal diversion in complex open pelvic fractures from blunt trauma. Arch Surg 1994;129:958-63.

15 Fitzgerald CA, Morse BC, Dente CJ. Pelvic ring fractures: has mortality improved following the implementation of damage control resuscitation? Am J Surg 2014:208:1083-90.

16 Velmahos GC. Pelvis. In: Mattox KL, Moore EE, Feliciano DV, Trauma. New York: McGraw-Hill Medical, 2013.

17 Stahel PF, Burlew CC, Moore EE. Current trends in the management of hemodynamically unstable pelvic ring injuries. Curr Opin Crit Care 2017;23:511-9.

18 Maull KI, Sachatello CR, Ernst CB. The deep perineal laceration - an injury frequently associated with open pelvic fractures. J Trauma 1977:17:685-96.

19 Woods RK, O'Keefe G, Rhee P, Routt ML, Maier RV. Open pelvic fracture and fecal diversion. Arch Surg 1998:133:281-6.

20 Pell M, Flynn WJ, Seibel RW. Is colostomy always necessary in the treatment of open pelvic fractures? J Trauma 1998:45:371-3. 